\title{
Correction to: Shannon information entropy sum of the confined hydrogenic atom under the influence of an electric field
}

\author{
S. J. C. Salazar ${ }^{1}$ (D), H. G. Laguna ${ }^{1, a}$ (D), B. Dahiya ${ }^{2}$, V. $\operatorname{Prasad}^{3}$ (D), and R. P. Sagar ${ }^{1}$ (D)
}

1 Departamento de Química, Universidad Autónoma Metropolitana, San Rafael Atlixco No. 186, Iztapalapa 09340, Ciudad de México, México

2 Department of Physics and Astrophysics, University of Delhi, Delhi 110007, India

3 Department of Physics, Swami Shraddhanand College, University of Delhi, Delhi 110036, India

Published online 24 September 2021

(C) EDP Sciences, SIF and Springer-Verlag GmbH Germany, part of Springer Nature 2021

Correction to: Eur. Phys. J. D (2021) 75:127

https://doi.org/10.1140/epjd/s10053-021-00143-2

The article was published with an error in equation 6 .

It should read:

The time-independent radial Schrödinger equation is written as

$$
\left[-\frac{\hbar^{2}}{2 m} \frac{d^{2}}{d r^{2}}-\frac{Z}{r}+\frac{\hbar^{2}}{2 m} \frac{l(l+1)}{r^{2}}\right] R_{n l}(r)=E_{n l} R_{n l} .
$$

This has been corrected in the original article. No other changes to the article have been made.

The original article can be found online at https://doi.org/ 10.1140/epjd/s10053-021-00143-2.

a e-mail: hlag@xanum.uam.mx (corresponding author) 